\title{
Spectroscopy of neutron-rich nuclei at REX-ISOLDE with MINIBALL
}

\author{
Th. Kröll for the MINIBALL and REX-ISOLDE collaborations \\ Institut für Kernphysik, Technische Universität Darmstadt, Darmstadt, Germany
}

\begin{abstract}
In this contribution, recent results obtained in nuclear structure studies with post-accelerated radioactive ion beams from the REX-ISOLDE facility at CERN are presented. The method employed is $\gamma$-ray spectroscopy with the MINIBALL array following "safe" Coulomb excitation and nucleon-transfer reactions.
\end{abstract}

\section{Introduction}

ISOLDE at CERN is the world-leading ISOL ("Isotope Separation OnLine") facility with more than 45 years of experience in producing low-energy radioactive ion beams (RIBs) [1]. So far, more than 1000 different isotopes have been delivered to experiments. The $1.4 \mathrm{GeV}$ proton beam from the PS Booster impinges on a thick production target, in most cases discussed in this contribution consisting of $\mathrm{UC}_{x} /$ graphite $\left(\approx 50 \mathrm{~g} / \mathrm{cm}^{2}{ }^{238} \mathrm{U}\right)$. Alternatively, the protons irradiate a Ta or $\mathrm{W}$ rod next to the production target and the generated neutrons induce the fission of the uranium. Compared to the direct proton impact, the chemical or isotopical composition of the fragments is changed. The produced nuclei diffuse within the heated target and reach the ion source. Here, a selection of the chemical element is intended. Sometimes it is possible to ionise only a certain isotope or even a single long-lived state of the nucleus. The highest selectivity is offered by the RILIS ("Resonant Ionisation Laser Ion Source"). The singly charged ions are extracted and sent through one of the two mass separators available at ISOLDE (GPS and HRS). In principle, the selection of the chemical element and the mass allows for isotopically pure beams. However, most of the beams contain a cocktail of different isobars because non-selective thermal ionisation is unavoidable. After passing the separator, a 30-60 keV low-energy beam can be sent to the experiments.

The idea of the REX-ISOLDE facility has been to post-accelerate these beams enabling nuclear reaction studies [2]. Firstly, the ions are cooled and bunched in a penning trap (REXTRAP). Then, they are sent to a second trap where the ions are charge bred, i.e. their charge state is increased by collisions with an intense electron beam (REX-EBIS). At a mass to charge ratio of about $A / q=4$, the ions are injected into a linac (REX-LINAC) where the energy is boosted to currently up to $3 \mathrm{MeV} / \mathrm{u}$. REX-ISOLDE operates now successfully for 10 years and post-accelerated ISOL beams of more than 80 isotopes of elements ranging from $\mathrm{Li}$ to $\mathrm{Ra}$ have been delivered to experiments.

The most important instrument for the study of exotic nuclei at REX-ISOLDE is the highly efficient MINIBALL spectrometer consisting of 8 triple clusters of six-fold segmented HPGe detectors [3]. MINIBALL was the first large $\gamma$-ray spectrometer equipped with fully digital electronics. The $\gamma$-rays are usually measured in coincidence with beam- and target-like nuclei detected by arrays of segmented $\mathrm{Si}$ detectors.

\section{Coulomb excitation}

Many experiments at REX-ISOLDE with MINIBALL employ $\gamma$-ray spectroscopy following "safe" Coulomb excitation as a tool to study collective properties of exotic nuclei. Here, the beam energy is chosen such that projectile and target interact only via the well-known electromagnetic interaction. The radioactive isotope of interest impinges as beam on a target and either of them (or both) can be excited. From the double-differential cross sections, extracted from the measured $\gamma$-ray-particle yields, the electromagnetic matrix elements are determined. As the cross section depends on both transitional 
but also diagonal matrix elements, reduced transition probabilities $B(E \lambda)$ as well as electric quadrupole moments $Q_{2}$ can be measured. In the analysis, the experimental data are compared with results of calculations solving a system of coupled differential equations to obtain the cross sections for a set of matrix elements. The use of a target nucleus with known properties allows for a analysis of the projectile excitation relative to the target excitation avoiding the necessity to determine absolute efficiencies and beam intensities. Other observables, like lifetimes or $\mathrm{g}$ factors, have been measured too by adapting methods used with stable beams to the requirements for experiments at REX-ISOLDE.

However, as isobaric contaminants in the beam excite the target as well the beam composition has to be determined. Different methods have been developed for this task comprising switching on/off the RILIS, stopping the beam inside MINIBALL and analysing the characteristic decay $\gamma$-rays, or using a $\Delta E-E$ telescope or a Bragg detector mounted in the beam dump downstream of MINIBALL.

One corner stone of the physics programme aims for the study of the evolution of collectivity near shell closures in exotic nuclei. Some key isotopes investigated at REX-ISOLDE are ${ }^{30,32} \mathrm{Mg}$ [4], ${ }^{68} \mathrm{Ni}[5]$, neutron-rich $\mathrm{Cu}$ isotopes [6-8], ${ }^{80} \mathrm{Zn}[9,10],{ }^{96} \mathrm{Kr}[11]$ and the region around ${ }^{132} \mathrm{Sn}$.

The isotope ${ }^{132} \mathrm{Sn}$ is the heaviest doubly-magic nucleus below ${ }^{208} \mathrm{~Pb}$ which is experimentally accessible. As the astrophysical $r$ process is expected to pass via this region, the understanding of the nuclear structure is also important for modelling the nucleosynthesis. At REX-ISOLDE, the Cd and Xe isotopical chains have been investigated. For the even isotopes ${ }^{138-144} \mathrm{Xe}$, the preliminary $B\left(E 2 ; 0_{\mathrm{gs}}^{+} \rightarrow\right.$ $2^{+}$) values obtained at REX-ISOLDE are well within the range of predictions by both a simple Grodzinstype systematics and theory calculations $[12,13]$. The same regular behaviour is observed for the $\mathrm{Ba}$ isotopes. Recently, it was possible by the combination of the cross section for Coulomb excitation and the lifetime from a DSAM measurement, both experiments were performed at REX-ISOLDE with MINIBALL, to determine the quadrupole moment of the first $2^{+}$state in ${ }^{140} \mathrm{Ba}$ [14].
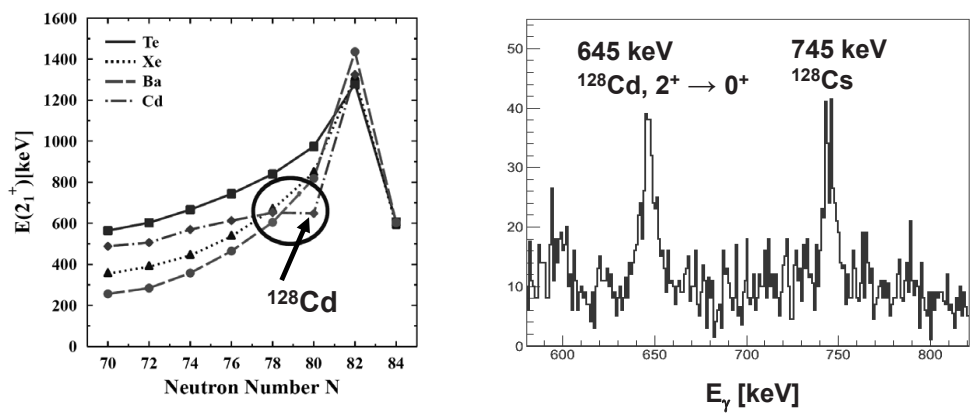

Fig. 1: Systematics of excitation energies $E\left(2^{+}\right)$around ${ }^{132} \mathrm{Sn}$ (left) and preliminary Doppler corrected $\gamma$-ray spectrum following "safe" Coulomb excitation of ${ }^{128} \mathrm{Cd}$ (right) [16]. An additional $\gamma$-ray at $745 \mathrm{keV}$ has been assigned as new transition to ${ }^{128} \mathrm{Cs}$, an isobaric contaminant in the beam.

Below $\mathrm{Sn}$, the Cd isotopes are of particular interest. The even isotopes ${ }^{122-128} \mathrm{Cd}$ and the odd ${ }^{123} \mathrm{Cd}$ have been investigated at REX-ISOLDE $[12,13,15,16]$. A special case is the isotope ${ }^{128} \mathrm{Cd}$, a short-lived nucleus $\left(T_{1 / 2}=280 \mathrm{~ms}\right)$ which is already 12 neutrons off stability. Although being nearer to the shell closure at $N=82$, the excitation energy of the first $2^{+}$state is lower than in the lighter neighbour ${ }^{126} \mathrm{Cd}$ (Fig. 1, left). This strikingly irregular behaviour cannot be explained by shell model calculations. Only beyond-mean-field calculations are able to reproduce the energy and predict a considerable deformation for this nucleus having only two protons and two neutrons less than the doubly-magic ${ }^{132} \mathrm{Sn}$ [17]. At REX-ISOLDE, the quadrupole collectivity of ${ }^{128} \mathrm{Cd}$ has been studied recently [16]. Fig. 1 
(right) shows the preliminary $\gamma$-ray spectrum obtained after Doppler correction with respect to the scattered $\mathrm{Cd}$ ions. The obtained $B(E 2)$ value will challenge the predictions from theory.

Recently, also very heavy nuclei became available as beams at REX-ISOLDE. E.g the phenomenon of shape coexistence has been investigated in light $\mathrm{Hg}$ and Po isotopes. Other important results obtained from Coulomb excitation concern the investigation of octupole collectivity in Rn and $\mathrm{Ra}$ isotopes with ${ }^{224} \mathrm{Ra}$ being the first isotope for which a $B(E 3)$ value has been measured at REXISOLDE [18].

\section{Transfer reactions}

In the last some years, one- and two-neutron transfer reactions became an important tool to study singleparticle properties at REX-ISOLDE. A dedicated set-up of segmented Si detectors with a large solid angle coverage, T-REX, has been constructed allowing to measure energy and position of light charged particles as well as to identify the species [19]. So far, $(d, p)$ and $(t, p)$ reactions in inverse kinematics have been studied with T-REX and MINIBALL.

The transfer data are analysed using the DWBA approach which is based on the use of empirical optical potentials. The observed cross section is described by the calculated cross section multiplied by a "spectroscopic factor" which is given by the overlap of the neutron(s) coupled to the inital state and the wave function of the final state. However, the concept of "spectroscopic factors" is highly debated. In practice, a problem is that the parameters of the optical potentials are not known for radioactive nuclei. They have to be extrapolated from parameter sets determined for stable beams or fitted to the elastic scattering measured in the actual experiment. We can conclude that the extracted cross sections in the experiments presented below depend only slightly on the actual choice. In Fig. 2, the experimental differential cross section for elastic scattering of ${ }^{30} \mathrm{Mg}$ at an energy of $1.8 \mathrm{MeV} / \mathrm{u}$ on protons and tritons is shown in comparison with results from DWBA calculations using different optical potentials [20].

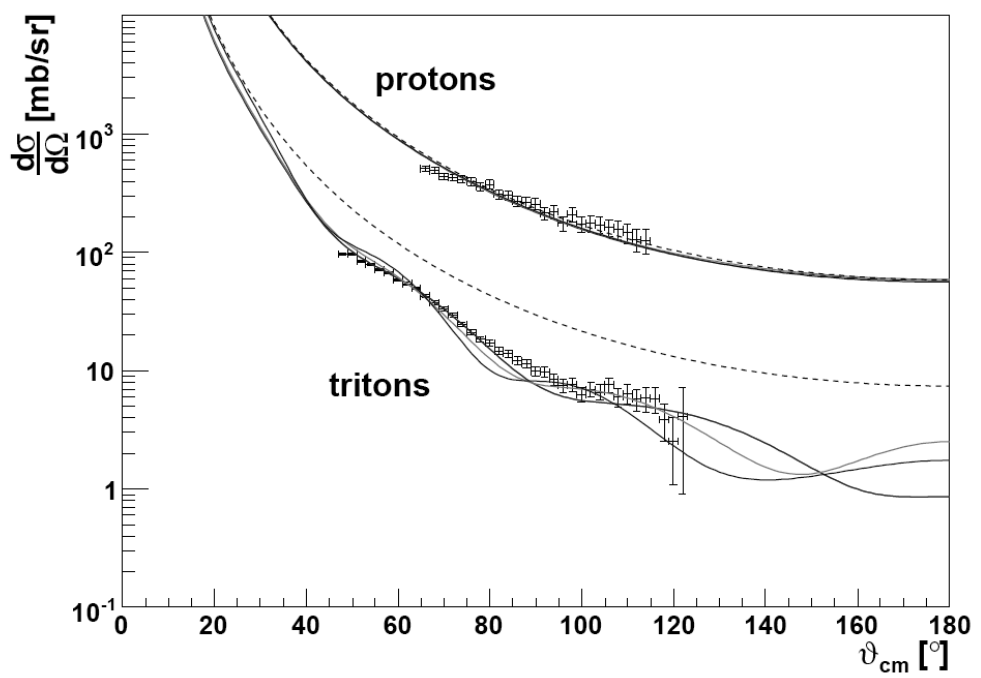

Fig. 2: Elastic scattering of ${ }^{30} \mathrm{Mg}$ on protons and tritons at $1.8 \mathrm{MeV} / \mathrm{u}$. Pure Coulomb potential (dashed lines) and extrapolated or fitted optical potentials (solid lines) [20].

One physics case studied in experiments performed at REX-ISOLDE was the investigation of 
the nuclear structure at the border of the "island of inversion", a region of the nuclear chart where the traditional shell closure at $N=20$ disappears. Excited states in the isotopes ${ }^{31,32} \mathrm{Mg}$ have been populated by $(\mathrm{d}, \mathrm{p})$ and $(\mathrm{t}, \mathrm{p})$ reactions in inverse kinematics [20-22].

Most prominent result was the discovery of the long-sought second $0^{+}$state in ${ }^{32} \mathrm{Mg}$. From most experimental data and theory calculations it has been concluded that in ${ }^{30} \mathrm{Mg}$ exists a coexistence between two $0^{+}$states with different shapes, the nearly spherical ground state and a deformed excited $0^{+}$state. Hence, ${ }^{30} \mathrm{Mg}$ is outside of the "island of inversion", see e.g. [4, 23]. For ${ }^{32} \mathrm{Mg}$, the situation is not so clear yet. The large $B\left(E 2 ; 0_{\mathrm{gs}}^{+} \rightarrow 2^{+}\right)$value indicates a deformed ground state with a $f p$ intruder configuration, hence the nearly spherical $s d$ configuration forming the ground state of ${ }^{30} \mathrm{Mg}$ is expected as an excited $0_{2}^{+}$state in ${ }^{32} \mathrm{Mg}$. However, such a state has never been observed experimentally before. At REX-ISOLDE, the population of an excited $0^{+}$state was unambiguously identified by the angular distribution of the protons following a (t,p) reaction on a ${ }^{30} \mathrm{Mg}$ beam which was characteristic for an orbital angular momentum transfer $\Delta L=0$. As the two-neutron transfer will populate only $0 p-0 h$ or $2 p-2 h$ configurations in ${ }^{32} \mathrm{Mg}$, the wave functions of the populated states will have the respective configurations and, consequently, large cross sections for the ground state $(2 p-2 h)$ and the $0_{2}^{+}$state $(0 p-0 h)$ are expected. Such an experiment became feasible because of the use of a tritium loaded Ti foil as target, the first radioactive target at REX-ISOLDE. The cross section for the population of the newly found $0^{+}$state is consistent with the assumption of a $s d$-configuration with a $p_{3 / 2}$ admixture, similar as the ground state of ${ }^{30} \mathrm{Mg}$. However, the low excitation energy remains a challenge for nuclear theory.

Further experiments performed during the last years are still under analysis. The nuclei investigated were ${ }^{12} \mathrm{Be},{ }^{46} \mathrm{Ar},{ }^{67,68} \mathrm{Ni}$, and ${ }^{79} \mathrm{Zn}$. Aim is to investigate the evolution of shell closures at $N=8,28,40$, and 50 .

Currently, transfer reactions are limited by the available beam energy to medium-mass nuclei up to $A=80$, because for heavier beams the angular distributions of the light products become more and more flat and featureless. This prevents to extract the transferred orbital angular momentum from the data. However, cross sections at low energy are of importance for astrophysical scenarios.

\section{Summary and Outlook}

The concept of the REX-ISOLDE facility at CERN, the post-acceleration of low-energy ion beams from ISOLDE, has been proven to be highly successful. With a broad range of isotopes at energies of up to $3 \mathrm{MeV} / \mathrm{u}$ available, many aspects and questions concerning the structure of exotic nuclei far-off the valley of stability have been addressed. Most important method is the $\gamma$-ray spectroscopy following "safe" Coulomb excitation and nucleon-transfer reactions. The experimental set-up consisting of the efficient high-resolution HPGe spectrometer MINIBALL in combination with arrays of segmented $\mathrm{Si}$ detectors is a powerful and versatile instrumentation to perform such studies.

In the near future REX-ISOLDE will be upgraded step-wise to HIE-ISOLDE [24]. By the end of 2014 , beam energies of up to $5.5 \mathrm{MeV} / \mathrm{u}$ will be available. In a second step, the energy will be increased further up to $10 \mathrm{MeV} / \mathrm{u}$. This is achieved by replacing the resonators of the REX linac with superconducting cavities. Additionally, higher beam intensities and an improved beam quality are envisaged.

The higher beam energy will allow to extend the physics programme considerably. In Coulomb excitation experiments with heavy targets, the probability for multi-step processes as well as for onestep excitation of high-lying states will be strongly enhanced. In particular, transfer reactions will profit as at HIE-ISOLDE such reactions can be studied also with heavy beams, some of them are unique to ISOLDE as low-energy beams. Such experiments aim at the investigation of single-particle properties in heavy nuclei. The investigation of the evolution of single-particle orbitals near shell closures will challenge modern shell model calculations. One aspect e.g. is the spin-orbit coupling in exotic nuclei which may be altered by a more diffuse surface of neutron-rich nuclei. Two-neutron transfer reactions 
are an excellent tool to populate shape-coexisting $0^{+}$states as well as to study pairing correlations far-off stability.

The success of REX-ISOLDE and the perspectives at HIE-ISOLDE have initiated also ideas for additional new instrumentation to be operated at HIE-ISOLDE like a helical orbit spectrometer, HELIOS, to study nucleon-transfer reactions. A major step will be the installation of the storage ring TSR [25]. It will allow for the study of reactions of stored exotic nuclei with an internal gas target.

These developments will extend the physics programme conducted at ISOLDE to new horizons and the study of exotic nuclei at CERN has a bright future.

\section{Acknowledgements}

This contribution represents the work of many groups, in particular PhD students and young postdocs, from all over Europe. The success of the REX-ISOLDE is owed to the large commitment of the ISOLDE and REX teams resulting in a continuous improvement of the facility.

The author acknowledges support by the German BMBF under grants 06MT238, 06DA9036I, 06DA7040, HIC for FAIR, EU through EURONS (No. 506065) and ENSAR (No. 262010) as well as the MINIBALL/REX-ISOLDE collaborations.

\section{References}

[1] http://isolde.web.cern.ch/ISOLDE/.

[2] D. Habs et al., Nucl. Instr. and Meth. B 139 (1998) 139; http://isolde.web.cern.ch/ISOLDE/ REX-ISOLDE/index.html.

[3] J. Van de Walle et al., Eur. Phys. J. A (in preparation).

[4] O. Niedermaier et al., Phys. Rev. Lett. 94 (2005) 172501.

[5] N. Bree et al., Phys. Rev. C 78 (2008) 047301.

[6] I. Stefanescu et al., Phys. Rev. Lett. 98 (2007).

[7] I. Stefanescu et al., Phys. Rev. Lett. 100 (2006) 112502.

[8] E. Rapisarda et al., Phys. Rev. C 84 (2011) 064323.

[9] J. Van de Walle et al., Phys. Rev. Lett. 99 (2007) 142501.

[10] J. Van de Walle et al., Phys. Rev. C 79 (2009) 014309.

[11] M. Albers et al., Phys. Rev. Lett. 108 (2012) 062701.

[12] Th. Kröll et al., INPC2007 proceedings, Nucl. Phys. A 805 (2008) 394.

[13] T. Behrens, PhD thesis (TU München, 2010).

[14] C. Bauer et al., Phys. Rev. C (in press).

[15] S. Ilieva et al., in preparation.

[16] S. Bönig et al., ISOLDE Workshop 2011.

[17] T. R. Rodríguez et al., Phys. Lett. B 668 (2008) 410.

[18] L. Gaffney, PhD Thesis (University of Liverpool, 2012); to be published.

[19] V. Bildstein et al., Eur. Phys. J. A 48 (2012) 85.

[20] K. Wimmer, PhD Thesis (TU München, 2010).

[21] K. Wimmer et al., Phys. Rev. Lett. 105 (2010) 252501.

[22] V. Bildstein, PhD Thesis (TU München, 2010); to be published.

[23] W. Schwerdtfeger et al., Phys. Rev. Lett. 103 (2009) 012501.

[24] http://hie-isolde.web.cern.ch/hie-isolde/.

[25] M. Grieser et al., Eur. Phys. J. Special Topics 207, (2012) 1. 